\title{
Visualization the dynamic interactive maps for results of spatio-temporal scanning
}

\author{
Xiaoxiao Song ${ }^{\star 1,2}$, Yan $\mathrm{Li}^{1,2}, \mathrm{Xia}_{\mathrm{XiaO}}{ }^{1,2}$, Le Cai ${ }^{1,2}$, Wei Liü ${ }^{1,2}$, Wenlong Cui ${ }^{1,2}$ and Ling Yue ${ }^{1}$ \\ ${ }^{1}$ School of Public Health, Kunming Medical university, Kunming, China; ${ }^{2}$ Yunnan Provincial Collaborative Innovation Center for Public \\ Health and Disease Prevention and Control, Kunming, China
}

\section{Objective}

The purpose of this article was to provide static and interact mapping for the results' SaTscan with R package thereby reduce the gap between decision-makers and researchers.

\section{Introduction}

Scan statistics is one of the most widely used method for detecting and locating the clusters of disease or health-related events through the space-time dimension. Although the Specific software of SatScan is available for free and easier to use graphical user interface (GUI) interface, the click way and the resulting text format have became obstacles in biosurveillance since automated or reproduction operation and the fast communicate information tool appeared. With the power of R software and 'rsatscan' package, we extended the visualization results to become a faster, more effective communication and motivation tool.

\section{Methods}

All the data are from a syndromic surveillance and real-time early warning system, which established 3 counties in the Yunnan province in the China for improving the ability to handle public health emergencies events and reduce the potential risk of disease spread. To illustrate the purpose of visualization, we only use one county data from 2017/9/1 to 2017/9/30 which includes two data sources: primary schools' absentees and health clinics' records. Based on the 'rsatscan' package which makes it easy to work within SaTscan from $\mathrm{R}$, we developed three ways for the results of Spatio-temporal scan: traditional tables, static maps and interactive visualize maps. Especial the last interactive visualization benefits from dynamic queries which may be an incredible tool to explore potential "clusters" data.

Data are collected from web-based by smart-phone or internet including 83 health clinics and 118 primary schools for one month. All the programs are run on Rstudio. The retrospective spatio-temporal scan parameters for two data sources as follow: Analysis type $=$ retrospective Space-Time, Analysis type $=$ Space-Time Permutation, Model Type $=$ High Rates (Poisson), Time precision=day, Time aggregation units $=$ day, MaxSpatialSizeInDistanceFromCenter $=10 \mathrm{~km}$, MaxTemporalSize $=14$ day.

\section{Results}

76211 records in the health clinics and 6066 absenteeism in the primary schools are collected. Three ways for the spatio-temporal scan results are provided in table (Tab-1), static maps (Fig 1) and interactive visualize maps online, some of them are presented in html format.

The table shows two data sources results by stack ways. The first column is the order of most likely to cluster. Follow is the code for center of the circular scan. The remaining indicators include Time Interval, Risk Value, Observed and expected value, P values from 999 Montel Carlo Simulation. See the table in more details.

The static maps have the advantages of vivid communicates information for where are the potential "cluster" both in two data sources over the space. What is more, one benefit of this way can provide the possible association between medical institution information and primary school absence information through the overlap circular.
The most excited is the interactive visualization with HTML format. From the click the navigate widgets on the left top, you can choose different layers. If you want to know more cluster information by the different potential cluster, clicking the map or dots or circular, and the pop-up dialogue box will show with the related clusters results of scan statistics methods.

See the detail in the website: http://rpubs.com/ynsxx/318257

\section{Conclusions}

These innovation ways can provide the ability to process information faster and to use that information to boost productivity and results. It is easy to help decision-makers to visualize communicates information faster than traditional reports. And the R code will more suitable for prospective analysis.

\begin{tabular}{|c|c|c|c|c|c|c|c|c|}
\hline luster & $0 c \_$ID & start_date & end_date & Risk & Observed_Num & pected_Num & P_value & data_type \\
\hline 1.00 & 12 & $2017 / 9 / 7$ & $2017 / 9 / 8$ & 9. 49 & 70.00 & 7.37 & 0.00 & Hospital \\
\hline 2.00 & 34 & $2017 / 9 / 23$ & $2017 / 9 / 30$ & 1. 73 & 624.00 & 360.85 & 0.00 & Hospital \\
\hline 3.00 & 8 & $2017 / 9 / 15$ & $2017 / 9 / 18$ & 6. 21 & 45.00 & 7. 25 & 0.00 & Hospital \\
\hline 4. 00 & 18 & $2017 / 9 / 1$ & $2017 / 9 / 3$ & 2. 07 & 179.00 & 86.48 & 0.00 & Hospital \\
\hline 5.00 & 83 & $2017 / 9 / 1$ & $2017 / 9 / 1$ & 10.72 & 24.00 & 2. 24 & 0.00 & Hospital \\
\hline 6.00 & 11 & $2017 / 9 / 4$ & $2017 / 9 / 10$ & 3. 04 & 79.00 & 25.95 & 0.00 & Hospital \\
\hline 7.00 & 42 & $2017 / 9 / 25$ & $2017 / 9 / 26$ & 21.56 & 15.00 & 0.70 & 0.00 & Hospital \\
\hline 8.00 & 63 & $2017 / 9 / 10$ & $2017 / 9 / 12$ & 2. 02 & 144.00 & 71.13 & 0.00 & Hospital \\
\hline 9. 00 & 33 & $2017 / 9 / 4$ & $2017 / 9 / 17$ & 1. 56 & 318.00 & 204.47 & 0.00 & Hospital \\
\hline 10.00 & 78 & $2017 / 9 / 4$ & $2017 / 9 / 10$ & 2. 71 & 71.00 & 26.24 & 0.00 & Hospital \\
\hline 11.00 & 9 & $2017 / 9 / 17$ & $2017 / 9 / 22$ & 3. 59 & 39.00 & 10.88 & 0.00 & Hospital \\
\hline 12.00 & 1 & $2017 / 9 / 16$ & $2017 / 9 / 24$ & 1. 72 & 156.00 & 90.47 & 0.00 & Hospital \\
\hline 13.00 & 50 & $2017 / 9 / 21$ & $2017 / 9 / 27$ & 5. 07 & 23.00 & 4. 53 & 0.00 & Hospital \\
\hline 14.00 & 73 & $2017 / 9 / 1$ & $2017 / 9 / 2$ & 10.00 & 13.00 & 1. 30 & 0.00 & Hospital \\
\hline 15.00 & 54 & $2017 / 9 / 15$ & $2017 / 9 / 19$ & 1. 71 & 101.00 & 59.05 & 0.00 & Hospital \\
\hline 16.00 & 32 & $2017 / 9 / 11$ & $2017 / 9 / 15$ & 1. 68 & 82.00 & 48.80 & 0.01 & Hospital \\
\hline 1.00 & 116 & $2017 / 9 / 2$ & $2017 / 9 / 3$ & 29.67 & 17.00 & 0.57 & 0.00 & School \\
\hline 2. 00 & 104 & $2017 / 9 / 10$ & $2017 / 9 / 10$ & 19. 75 & 14.00 & 0.71 & 0.00 & School \\
\hline 3.00 & 2 & $2017 / 9 / 6$ & $2017 / 9 / 7$ & 3. 77 & 40.00 & 10.60 & 0.00 & School \\
\hline
\end{tabular}

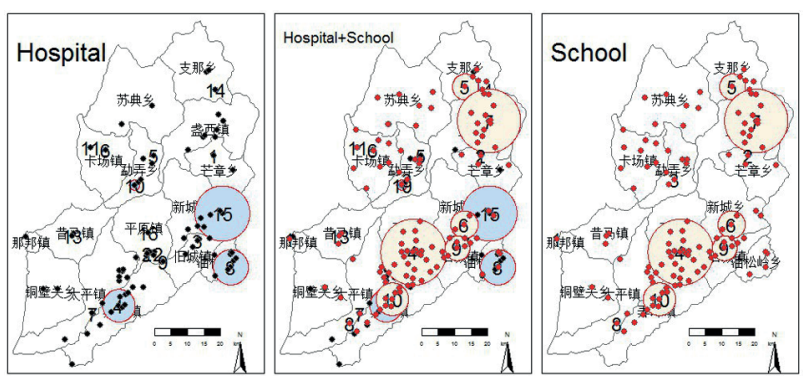

\section{Keywords}

Visualization; spatio - temporal scan; package 'rsatscan'

\section{References}

Kleinman K, R package 'rsatscan', 2015.

\section{*Xiaoxiao Song}

E-mail: chinasxx@gmail.com 